M. Kolb M. Schmidt

\section{Die Bedeutung von Zytokinen und Wachstums- faktoren bei fibrosierenden Lungenerkrankungen}

\author{
The Role of Cytokines and Growth Factors in Fibroproliferative Lung Disease
}

\section{Zusammenfassung}

Ein neues pathogenetisches Konzept zur Fibrogenese hat die Hypothese aufgestellt, dass es sich bei der Fibrose um „pathologische Wundheilung“ oder gestörtes „Remodeling“ handelt und Entzündungsvorgänge sekundär sind. Zytokine sind bei diesem Prozess maßgeblich involviert und ein attraktives Ziel für pharmakologische Interventionen. TGF $\beta$ hat als profibrotischer Wachstumsfaktor eine Schlüsselstellung und kann auf verschiedenen Wegen inaktiviert oder in seiner Wirkung abgeschwächt werden. Diese Übersichtsarbeit erläutert die Grundlagen der pathobiologischen Abläufe und stellt ihre potenzielle klinische Bedeutung dar.

\section{Abstract}

A new hypothesis of the pathogenesis of fibroproliferative lung disease suggests that fibrosis is caused by abnormal and excessive wound healing and pathologic tissue remodelling. Inflammation is possibly an epiphenomenon. Cytokines are critical players in the pathologic process and attractive targets for pharmacological intervention. TGF $\beta$ is a key profibrotic growth factor, a variety of approaches are known to modify and inhibit its activity. This article reviews the basic pathological concepts of pulmonary fibrogenesis and outlines its potential clinical benefit.

\section{Einleitung}

Fibrosierende Lungenerkrankungen sind Krankheiten, die sich im Alveolarraum und Interstitium abspielen und bei denen es durch überschießende Narbenbildung zu einer progredienten Destruktion von Gewebe mit irreversiblem Funktionsverlust kommt. Es gibt eine Reihe von bekannten Auslösern für die Erkrankung, mit einem weiten Spektrum von Mikroorganismen und inhalativen Noxen (z.B. Asbest, Silikate) über Medikamente (z.B. Bleomycin, Amiodaron) bis zu ionisierenden Strahlen und
Autoimmunerkrankungen (z. B. Sklerodermie, chronische Polyarthritis oder Dermatomyositis) [1,2]. In etwa 30-40\% der Fälle ist die Ätiologie der progressiven Fibrosierung jedoch unklar, man spricht dann von idiopathischer interstitieller Pneumonie. Es gibt unterschiedliche Subgruppen: UIP (Usual Interstitial Pneumonia), NSIP (Non-specific Interstitial Pneumonia), DIP (Desquamative Interstitial Pneumonia) und AIP (Acute Interstitial Pneumonia). Nach internationalem Konsensus sollte der Begriff idiopathischer Lungenfibrose (IPF) nur mit dem Typ UIP gleichgesetzt werden $[3,4]$. 


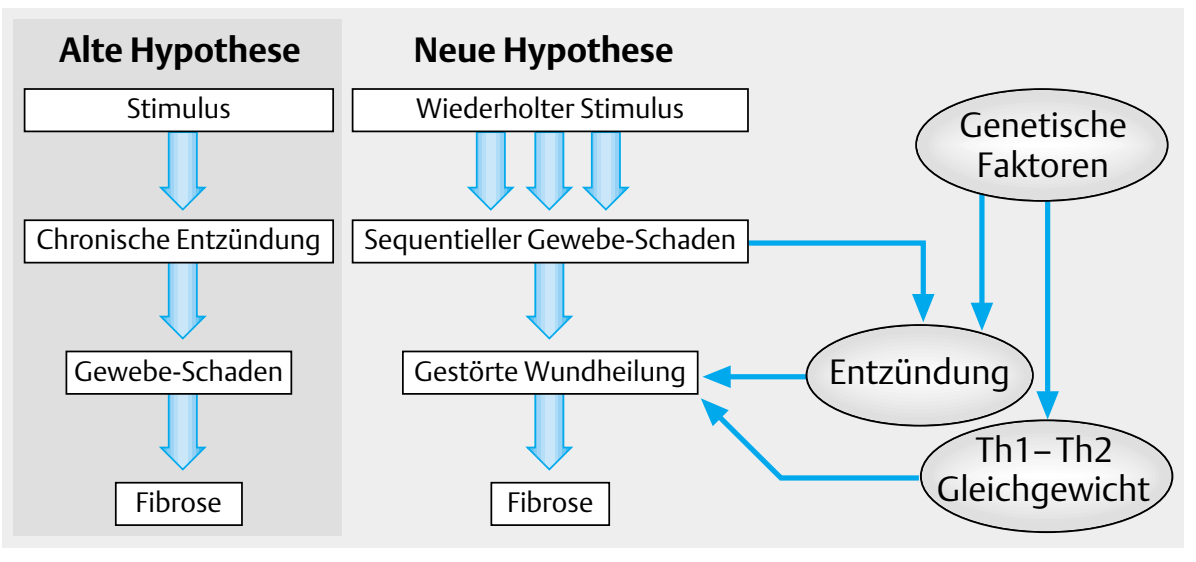

Abb. 1 Konzept der Pathogenese idiopathischer interstitieller Lungenfibrosen [2].

Die Angaben zur Inzidenz der IPF sind uneinheitlich, sie dürfte jedoch im Bereich von 10-30/100 000 liegen. Meist sind Patienten mittleren Alters betroffen (zwischen 40. und 70. Lebensjahr). Die Krankheit verursacht relativ rasch eine restriktive Ventilationsstörung mit Diffusionsstörung, und führt schließlich über respiratorische Insuffizienz oder komplizierende pulmonale Infektionen zum Tod. Die mittlere Überlebensrate nach Diagnosestellung ist zwei bis drei Jahre [2]. Die therapeutischen Optionen sind bescheiden. Dies könnte kaum besser zum Ausdruck gebracht werden als durch die Tatsache, dass Kortikosteroide kombiniert mit Azathioprin oder Cyclophosphamid als momentaner „Goldstandard“ der Therapie lediglich Ansprechraten zwischen 15 - $30 \%$ aufweisen [4].

\section{Pathogenese der Lungenfibrose}

Früher dachte man, dass eine chronische Entzündung im Alveolar- und interstitiellen Raum das Gewebe zerstört und durch wiederholte Reparation exzessive Narbenbildung und Fibrose verursacht [5]. Im Gegensatz dazu heilen akute Alveolitiden, wie z.B. im Rahmen des ARDS (Acute Respiratory Distress Syndrome), meist ohne größere Defekte aus, sofern die kritische Krankheitsphase überlebt wird [6]. Die neuen pathogenetischen Konzepte zur Fibrogenese beschreiben die Fibrose als „pathologische Wundheilung“ oder gestörtes „Remodeling“: wiederholte Entzündungen im mikroskopischen Bereich, gestörte Kommunikation zwischen Entzündungs- und strukturellen Zellen der Lunge und fokale Fibroblastenproliferation resultieren in überschießender Deposition und reduziertem Abbau von extrazellulärer Matrix (EZM) $[5,7,8]$. Ein wesentlicher pathogenetischer Faktor sind Dysregulationen auf der Ebene der Zytokine, da über diese Botenstoffe ein Großteil der interzellulären Kommunikation abläuft $[9,10]$. Bei IPF wird sogar diskutiert, dass die entzündliche Komponente lediglich die Krankheit triggert oder gar ein sekundäres Phänomen ist, und das pathologische Verhalten von Epithelzellen und Fibroblasten im Mittelpunkt des Problems steht $[5,7]$ [siehe Abb. 1].

\section{Zytokine als Vermittler zwischen Immunantwort und Wundheilung}

Immunantwort und interzelluläre Kommunikation werden überwiegend durch Zytokine vermittelt (siehe auch Tab.1). Es gibt proinflammatorische Zytokine, welche die Entzündungsreaktion fördern und zur verstärkten Einwanderung von Granulo- und Lymphozyten in das Wundgebiet führen, so z. B. TNF $\alpha$, IL-1, IL-6, IL-8, MCP-1 und MIP-1 [11]. Und es gibt antiinflammatorische Zytokine wie PDGF, FGFs, TGF $\alpha$ und TGF $\beta_{1_{-3}}$, mit denen der akute Prozess eingedämmt und die Gewebsregeneration eingeleitet und gesteuert werden [12-14]. Eine vereinfachende Trennung in diese zwei Gruppen hilft dem Verständnis der komplexen Regulationsvorgänge, entspricht aber nicht der Realität. Ein ausgewogenes Zusammenspiel „destruktiver“ und „konstruktiver“ Zytokine ist zur Erhaltung der Organintegrität nötig [15].

\section{Proinflammatorische Zytokine}

Mit wenigen Worten beschrieben dient der biologische Zweck akuter Entzündung der möglichst raschen Elimination schädlicher Einflüsse auf den Organismus. Proinflammatorische Zytokine steigern die Aktivität ortsständiger Entzündungszellen und wirken chemotaktisch auf Zellen aus der Zirkulation [15]. Vorübergehend fördern sie ein matrix-abbauendes Milieu, damit die Migration der Zellen im Gewebe erleichtert wird. IL-1, IL-6, IL-8

Tab. 1 Übersicht über Zytokine und Wachstumsfaktoren, denen in der Pathogenese fibrosierender Lungenerkrankungen eine gewisse Rolle zugeordnet wird. TGF $\beta$ und PDGF sind Faktoren mit besonderer Bedeutung und Zielmoleküle neuer pharmakologischer Wirkstoffe (siehe Text)

\begin{tabular}{|ll}
\hline IL-1 $\beta,-6,-8$ & Interleukine \\
\hline MCP-1 & Macrophage Chemotactic Protein \\
\hline MIP & Macrophage Inflammatory Protein \\
\hline$T N F \alpha$ & Tumor Necrosis Factor \\
\hline TGF- $\beta /-\alpha$ & Transforming Growth Factor \\
\hline PDGF & Platelet Derived Growth Factor \\
\hline CTGF & Connective Tissue Growth Factor \\
\hline FGF-1 und -2 & Fibroblast Growth Factor \\
\hline KGF & Keratinocyte Growth Factor \\
\hline GM-CSF & Granulocyte Macrophage Colony Stimulating Factor \\
\hline
\end{tabular}


und TNF $\alpha$ gehören zu den „klassischen“ proinflammatorischen Zytokinen und sind bei vielen akuten Entzündungsreaktionen in der Lunge präsent. Bei chronischen Immun- und fibroproliferativen Erkrankungen der Lunge scheinen zumindest IL-1 und TNFa eine Rolle zu spielen und werden mit der Fibrogenese assoziiert [16].

\section{Interleukin-1}

IL-1 liegt in zwei Isoformen mit gleichem Wirkprofil vor: IL-1 $\alpha$ ist membrangebunden und IL- $1 \beta$ findet sich in der Zirkulation [17]. In der Lunge sind fast alle Zellen zur Synthese von IL-1 $\beta$ befähigt, der Hauptbildungsort sind aktivierte Makrophagen. IL-1 ist ein vielseitiges proinflammatorisches Zytokin, das synergistisch zu TNF $\alpha$ wirkt [18]. IL-1 kann profibrotisch sein und Fibroblasten zur Synthese von Glycosaminoglykanen und Fibronectin, und auch zur Bildung weiterer Zytokine, u. a. PDGF und TGF $\beta$, stimulieren $[6,15,19]$. IL-1 kann aber auch ein degradierendes Umfeld erzeugen durch Induktion von Kollagenasen, Prostaglandin und Plasminogen-Aktivator [17]. In Tierversuchen wurde gezeigt, dass nach Anwendung von Bleomycin, Silikat und ionisierenden Strahlen IL-1 in den frühen Phasen nach Initiierung des Alveolarschadens hochreguliert ist [6,20-23]. Wenn man in die Rattenlunge einmalig rekombinantes IL-1 injiziert, sieht man eine akute neutrophile Entzündung mit Alveolar- und Kapillarschäden ähnlich wie beim ARDS [24,25]. Nach zweiwöchiger Überexpression von IL-1 $\beta$ durch transienten Gentransfer in die Lunge entwickelt sich nach Abklingen der akuten Entzündung eine progressive Fibrosereaktion, am ehesten vermittelt durch TGF $\beta$ [26].

Beim Menschen wurden erhöhte Werte von IL-1 in der bronchoalveolären Lavage-Flüssigkeit (BALF) und in Alveolarmakrophagen von Patienten mit ARDS, aber nicht IPF gefunden [6]. Es wurde auch in IPF Gewebe in myofibroblastenreichen Arealen nachgewiesen [22,27]. Genetische Studien lassen vermuten, dass IL-1 Rezeptor-Antagonist Polymorphismen häufiger bei IPF-Patienten zu finden sind, ein möglicher Hinweis auf die Bedeutung eines gestörten Gleichgewichtes zwischen IL-1 und seinen Regulatoren [27].

\section{TNF $\alpha$}

TNF $\alpha$ wird in der Lunge nach Stimulation rasch von Alveolarmakrophagen und Typ-II-Pneumozyten freigesetzt [9]. TNF $\alpha$ fördert die Migration und Adhäsion von Granulozyten und Monozyten, die Freisetzung von Sauerstoffradikalen und zytolytischen Faktoren, löst eine Zytokin-Kaskade aus und reguliert Apoptose [28]. Weiterhin aktiviert TNF $\alpha$ mesenchymale Zellen und beeinflusst den Kollagenstoffwechsel, sowohl in pro- als auch antifibrotischer Weise [9,29]. Zahlreiche Tierstudien haben gezeigt, dass TNF $\alpha$ bei akuter und chronischer Gewebsschädigung nach Einwirkung von Bleomycin, Asbest, Silikat und ionisierenden Strahlen beteiligt ist [9]. TNF $\alpha$-Rezeptor-knockout-Mäuse entwickeln nach Bleomycin-, Asbest- oder Silikatinjektion eine mildere Lungenfibrose als Wildtypen [30]. Wenn man in der Lunge TNF $\alpha$ durch Gentransfer überexprimiert, kommt es zu einer schweren akuten Entzündungsreaktion, gefolgt von verhältnismäßig milden Parenchymvernarbungen [31]. Die profibrotischen Effekte von TNF $\alpha$ werden durch TGF $\beta$ und PDGF vermittelt [9].
TNF $\alpha$ wird in BAL-Makrophagen von Patienten mit IPF und Asbestose verstärkt exprimiert [9]. In BAL-Zellen von Patienten mit IPF, Bronchiolitis obliterans mit organisierter Pneumonie (BOOP) und Sarkoidose kann TNF $\alpha$ hochreguliert [32] oder normal sein [9].

\section{Profibrotische Zytokine}

Für die Integrität eines biologischen Systems ist es wichtig, dass Intensität, örtliche Ausdehnung und Dauer einer entzündlichen Reaktion auf das nötige Maß beschränkt sind. Durch antiinflammatorische und profibrotische Zytokine versucht das Immunsystem, die Entzündung einzudämmen und Schäden zu reparieren. Wenn das misslingt, können ausgedehnte Gewebsdefekte entstehen. Sind die Mechanismen überaktiv, kann es zu „exzessiver Wundheilung" kommen mit der Konsequenz irreversibler Gewebsfibrose $[7,8]$. Zahlreiche Wachstumsfaktoren sind in dem Prozess beteiligt, besonders wichtig sind TGF $\beta$, PDGF und FGFs.

\section{Transforming Growth Factor}

Drei TGF $\beta$-Isoformen werden in menschlichen Zellen gefunden, es ist ein pluripotentes Zytokin mit breitem Wirkspektrum. Die Isoform TGF $\beta 1$ kommt am häufigsten vor und beeinflusst den Metabolismus extrazellulärer Matrix (EZM) und Stromazell-Aktivität [33]. Die immunomodulatorischen Aktivitäten von TGF $\beta$ sind lebensnotwendig und limitieren u.a. akut-entzündliche Prozesse [34]. Die meisten Zellen können TGF $\beta$ bilden, in der Lunge sind es v.a. Alveolarmakrophagen, Epithelzellen und Fibroblasten. Das Molekül wird in inaktiver Form sezerniert und durch oxidative, proteolytische oder integrinvermittelte Abspaltung des „latency associated peptide“ (LAP) aktiviert $[9,34,35]$. TGF $\beta$ stimuliert die Synthese von EZM, insbesondere Kollagen und Fibronectin, und vermindert den Matrixabbau durch Änderung des Gleichgewichtes von Kollagenasen (Matrix Metalloproteinasen - MMP) und Kollagenase-Inhibitoren (tissue inhibitor of metalloproteinase - TIMP) [33]. Es ist antiproliferativ für Epithelzellen, ein wichtiger Aspekt bei pathologischem Gewebs-Remodeling [36], und fördert die Transformation von Fibroblasten zu Myofibroblasten [12]. TGF $\beta$ kann weiterhin profibrotische Faktoren wie FGF, PDGF und CTGF induzieren [37]. Die Expression von TGF $\beta$ wird durch TNF $\alpha$, IL-1, GM-CSF, PDGF und TGF $\beta$ gesteigert. TGF $\beta$ gilt als ein Schlüssel-Zytokin fibrosierender Erkrankungen. Zahlreiche experimentelle Modelle und immunhistologische Untersuchungen an humanem Gewebe haben das bei Lungen-, Leber- und Nierenfibrose gezeigt $[33,38]$.

TGF $\beta$ ist in allen Modellen der Lungenfibrose nachweisbar und an der Progression der Gewebsreaktion beteiligt $[9,39]$. Wenn man TGF $\beta 1$ durch Gentransfer in Rattenlungen überexprimiert, kommt es nach einer Woche zur Entwicklung einer progressiven Lungenfibrose und zwar ohne wesentliche Entzündung [40]. Die Progression der Lungenfibrose nach Transfer des IL-1 $\beta$ oder TNF $\alpha$-Gens wiederum ist abhängig von der Induktion von endogenem TGF $\beta$ (Tab. 2). Es gibt umfangreiche Hinweise, dass TGF $\beta$ bei fibroproliferativen Lungenerkrankungen des Menschen beteiligt ist [9]. TGF $\beta$ wird in Gewebe, BAL-Zellen und -Flüssigkeit von Patienten mit Silikose, Asbestose, UIP, progressiver Sarkoidose, Sklerodermie und Strahlenfibrose gefunden $[9,41]$. 
Tab. 2 Effekt der Überexpression von Zytokinen auf die Lunge (durch transienten Gen-Transfer). Die proinflammatorischen Zytokine können keine (IL-8), leichte (TNF $\alpha$ ) und schwere (IL-1 $\beta$ ) fibrotische Veränderungen induzieren. Die Ausprägung scheint von der Anwesenheit und Konzentration von TGF $\beta$ in der Lunge abzuhängen. ( $\alpha$ SMA ist eine immunhistochemische Methode und weist auf das Vorhandensein von Myofibroblasten hin)

\begin{tabular}{lllll}
\hline Zytokin & Entzündung & TGF $\beta$ (BALF) & $\alpha$ SMA & Fibrose \\
\hline IL-8 & akut ++++ & nicht messbar & - & - \\
TNF $\alpha$ & akut +++ & $150 \mathrm{pg} / \mathrm{ml}$ & + & + \\
IL-1 $\beta$ & $\begin{array}{l}\text { akut }+++ \\
\text { alveoläre Destruktion }\end{array}$ & $400 \mathrm{pg} / \mathrm{ml}$ & +++ & ++++ \\
TGF $\beta 1$ & $(+)$ & $>50 \mathrm{ng} / \mathrm{ml}$ & ++++ & ++++ \\
\hline
\end{tabular}

\section{PDGF}

PDGF ist ein Glykoprotein-Dimer und wird in der Lunge von Alveolarmakrophagen, Epithel-, Endothelzellen und Fibroblasten gebildet [42]. Die Signaltransduktion erfolgt durch Tyrosinkinasen, ein wichtiges pharmakologisches Zielmolekül [43]. PDGF ist chemotaktisch für Fibroblasten, neutrophile Granulozyten und Makrophagen/Monozyten und kann die Fibronectin- und Prokollagen-Synthese steigern. PDGF induziert die Expression von TGF $\beta$, aber genauso wird die Expression von PDGF und PDGF-Rezeptoren von TGF $\beta$, IL-1 $\beta$, TNF $\alpha$ und bFGF induziert [9]. In Tierversuchen mit Asbest wurde gezeigt, dass in Bronchialaufzweigungen mit fibroproliferativen Läsionen PDGF und PDGFRezeptoren hochreguliert sind [44]. Alveolarmakrophagen und Fibroblasten exprimieren nach Stimulation mit Asbestfasern PDGF. PDGF-knockout-Mäuse sterben am Lungenemphysem, das sich wegen fehlender Myofibroblasten und pathologischer Alveolenreifung ausbildet [45].

Es gibt eine Reihe von Hinweisen, dass PDGF bei fibrosierenden Lungenerkrankungen des Menschen eine Rolle spielt. Bei IPF, Sklerodermie assoziierter Lungenfibrose und BOOP nach Lungentransplantation wurde beschrieben, dass PDGF-mRNA bzw. -Protein in Alveolarmakrophagen und Fibroblasten im Vergleich zu Gesunden gesteigert sein kann [9].

\section{FGF}

Zu den FGFs werden neun heparinbindende Peptide gezählt, von denen azidischer und basischer FGF (jetzt FGF-1 und -2) sowie KGF (jetzt FGF-7) für das Remodeling wichtig sind. FGF-1 und -2 fördern die Bildung von Granulationsgewebe, Fibroblasten-Proliferation und Kollagensynthese [37]. Sie haben Einfluss auf Angiogenese und Epithelzellregeneration, beides bedeutende Schritte bei der Wundheilung [46]. Im Tiermodell wird FGF-1 und -2 nach Injektion von Bleomycin oder Paraquat in die Lunge hochreguliert [47,48]. FGF-2 ist in BALF und Serum von Patienten mit IPF und Sklerodermie erhöht [49]. KGF ist ein Wachstumsfaktor für Typ-II-Pneumozyten [50]. Möglicherweise spielt KGF eine protektive Rolle im Prozess der Wundheilung. Bei Patienten mit ARDS wurde gezeigt, dass die Konzentration von KGF in der BALF ein Marker für die Schwere der Erkrankung sein kann und eventuell mit der Prognose korreliert [50].
Potenzielle Bedeutung für die Klinik

Die Erforschung der Pathophysiologie der Lungenfibrosen hat nicht nur grundlagenwissenschaftliche Bedeutung, sondern auch potenzielle klinische Relevanz. Zytokine und Wachstumsfaktoren sind in der Regel gut quantifizierbare Moleküle, die in Blut und anderen Körperflüssigkeiten wie z.B. BALF nachgewiesen werden können und eventuell als diagnostische Marker verwendet werden können. Die gezielte pharmakologische Inhibition von Zytokinen wird bereits bei einer Reihe von immunologischen Erkrankungen praktiziert.

\section{Zytokine als diagnostisches Mittel}

Es wurde bereits darauf hingewiesen, dass in menschlichem Lungengewebe eine Vielzahl von Zytokinen und Wachstumsfaktoren nachgewiesen werden kann, was ihre pathophysiologische Bedeutung unterstreicht. Molekularbiologische Gewebsuntersuchungen sind derzeit meist nur bei speziellen Fragestellungen für die klinische Arbeit entscheidend. Damit ein biochemisches Produkt als diagnostischer Marker breite klinische Relevanz erlangen kann, sollte man in der Lage sein, dieses Produkt zu quantifizieren und mit Normalwerten zu vergleichen. So wurde z.B. TGF $\beta$ im Plasma von Frauen mit Mammakarzinom gemessen, die im Rahmen einer Knochenmarkstransplantation einer Hochdosis-Chemotherapie und Radiatio unterzogen wurden. Die gemessenen Spiegel korrelierten mit dem Auftreten interstitieller Pneumonitiden [51], so dass die Bestimmung von TGF $\beta$ eventuell prädiktiven Wert für diese Komplikation erlangen könnte. Wir haben in eigenen Untersuchungen zeigen können, dass bei UIP im Vergleich zu DIP/NSIP, Sarkoidose und Kontrollpatienten erhöhte BAL-Konzentrationen von TGF $\beta$ vorhanden sind [52]. Diese Untersuchungen müssen aber noch bestätigt und validiert werden, von einem Einsatz in der Routine ist man noch weit entfernt.

Inhibition der proinflammatorischen Faktoren TNF $\alpha$ und IL-1 Man hat zeigen können, dass die Bleomycin- und Silikat-Fibrose durch lösliche TNF $\alpha$-Rezeptoren und IL-1-Rezeptor-Antagonist günstig beeinflusst werden kann [53,54]. Pentoxifyllin soll seine antifibrotische Wirkung teilweise über TNF $\alpha$ Inhibition entfalten [55]. Eine klinische Studie hat bei chronischer Sarkoidose einen positiven Effekt auf den Verlauf der Erkrankung gezeigt, wobei nicht unterschieden werden kann, ob dies durch Modulation des Immunsystems oder der Fibrogenese bedingt ist [56]. Auch für die neue antifibrotische Substanz Pirfenidon bestehen experimentelle Hinweis auf eine Wechselwirkung mit TNF $\alpha$ [57]. Monoklonale TNF $\alpha$-Antikörper und lösliche Rezeptoren sind zur Therapie der Rheumatoiden Arthritis und chronisch entzündlicher Darmerkrankungen zugelassen und haben sich bewährt, ebenso werden hier IL-1 Antagonisten eingesetzt [58]. Studien zur Wirksamkeit bei interstitiellen Lungenerkrankungen wurden mit diesen Medikamenten noch nicht durchgeführt. Es bestehen somit einige Möglichkeiten, TNF $\alpha$ und IL-1 in vivo $\mathrm{zu}$ hemmen, und es gibt experimentelle Daten, die einen klinischen Einsatz unterstützen. Man darf aber nicht vergessen, dass die prokollagenolytische Aktivität von TNF $\alpha$ und IL-1 in einem fortgeschritteneren Stadium der Lungenfibrose eventuell einen protektiven Charakter haben und die Zytokin-Blockade einen gegenteiligen Effekt haben könnte wie erhofft. Außerdem kann die Antagonisierung von TNF $\alpha$ und IL-1 zu Immunschwäche mit 
schwerwiegenden pulmonalen und systemischen Infektionen führen [9].

\section{Inhibition der profibrotischen Faktoren TGF $\beta$ und PDGF}

Die zentrale Stellung von TGF $\beta$ in der Pathogenese fibrosierender Lungenerkrankungen hat zur Entwicklung von Wirkstoffen mit Anti-TGF $\beta$-Effekt geführt. Einige Wirkstoffe blockieren TGF $\beta$ direkt (z.B. Antikörper oder lösliche Rezeptoren) oder binden ihn im Gewebe und verhindern so den Kontakt mit dem Rezeptor (z.B. Decorin) $[38,59-61,62]$. Andere Substanzen scheinen in noch unbekannter Weise mit der Expression, Synthese und/oder Freisetzung von TGF $\beta$ zu interferieren. Dazu gehören z.B. ACEHemmer, Interferon- $\gamma$, Niacin, Taurin oder Pirfenidon [63-66]. Ein Teil des reno- und kardioprotektiven Effektes von ACE-Hemmern bei chronischer Herz- und Niereninsuffizienz liegt an ihrer antifibrotischen Wirkung [67]. Es ist wahrscheinlich, dass diese teilweise über TGF $\beta$ vermittelt werden [68]. Angiotensin-II ist ein potentes Mitogen für Fibroblasten in vitro [68]. In Modellen der strahleninduzierten Nieren- und Lungenfibrose reduzierten sowohl die ACE-Hemmer Captopril und Enalapril als auch Angiotensin-II-Rezeptor-Antagonisten die Fibrosereaktion [63].

Auch die Inhibition von PDGF zur Therapie der Lungenfibrose wird in experimentellen Studien untersucht. Durch Gentransfer eines PDGF-Rezeptors, dem die intrazelluläre Tyrosinkinase fehlt, konnte die bleomycininduzierte Lungenfibrose verhindert werden [69].

\section{Erste klinische Daten zu Interferon- $\gamma$ 1b, Pirfenidon und Relaxin}

Interferon- $\gamma 1 \mathrm{~b}$, Pirfenidon und Relaxin sind neuere antifibrotische Substanzen, zu denen erste klinische Studien vorliegen [2].

Interferon- $\gamma$ hemmt die Proliferation von Lungenfibroblasten und ihre Proteinbiosynthese [70]. Im Bleomycin-Modell reduziert Interferon- $\gamma$ die Expression von TGF $\beta$, Prokollagen-I und -III [71]. Weiterhin ist denkbar, dass Interferon- $\gamma$ sich durch Hemmung einer Th2-gerichteten Immunantwort positiv auf die Fibrogenese auswirkt [2]. In einer randomisierten Studie wurden insgesamt 18 Patienten mit progressiver IPF eingeschleust und Prednisolon plus Interferon- $\gamma 1$ b s.c. gegen Prednisolon alleine untersucht [66]. Nach einem Jahr hatten sich die Lungenfunktionen und Sauerstoffpartialdrücke der Interferon-Patienten signifikant gebessert, die der Prednisolon-Gruppe hingegen leicht verschlechtert. Die vor Behandlung deutlich induzierte Genexpression von TGF $\beta$ und CTGF im Lungengewebe sank im Verlauf der Interferon-, aber nicht der Prednisolon-Therapie. Diese viel versprechenden Ergebnisse wurden einer Reanalyse durch ein Expertengremium unter Leitung von G. Raghu unterzogen und sind auf der Homepage der American Thoracic Society veröffentlicht (www.thoracic.org). Erste vorläufige Resultate einer multizentrischen, prospektiven Studie mit etwa 300 IPF-Patienten wurden auf dem Kongress der European Respiratory Society 2002 in Stockholm und auf dem International Colloquium on Lung Fibrosis in Glion, Schweiz, in Vorträgen vorgestellt. Nach 48 Wochen Behandlungszeit mit Interferon- $\gamma 1 \mathrm{~b}$ ergaben sich keine Unterschiede zu Plazebo bezüglich der primären StudienEndpunkte (Spirometrische Parameter und $\mathrm{pO}_{2}$ ). Ein nicht signifikanter Trend zu einem leichten Überlebensvorteil der Interferon- $\gamma 1 \mathrm{~b}-$ Gruppe nach diesem Zeitraum scheint durch Patienten in früheren Krankheitsstadien (FVC predicted $>60 \%$ ) bedingt zu sein, die möglicherweise am meisten von dieser Therapie profitieren könnten (T. King und G. Raghu, persönliche Mitteilung). Ob und wenn ja, für welche Patienten dieses Medikament sinnvollerweise (auch unter dem Gesichtspunkt der Kosten-Nutzen Relation) eingesetzt werden kann, bleibt abzuwarten und ist in weiteren kontrollierten Studien zu prüfen.

Pirfenidon ist eine antifibrotische Substanz, die In-vitro-Proliferation, Kollagensynthese und Matrixsekretion von Fibroblasten hemmt [2]. Das therapeutische Potenzial von Pirfenidon wurde im Bleomycin-Modell gezeigt, in dem es die Genexpression von TGF $\beta$ und Prokollagen reduzierte [57]. In einer Phase-II-Studie an Patienten mit fortgeschrittener IPF wurde Pirfenidon gut vertragen, die Lungenfunktionsparameter erschienen stabilisiert [72]. Randomisierte Studien sind in Planung.

Relaxin ist ein Stoff, der normalerweise vom Corpus luteum gebildet wird und die Symphyse und Zervix im Rahmen der Geburtsvorbereitung weich macht [67]. Relaxin hemmt die TGF $\beta$ induzierte Kollagensynthese in vitro, wirkt in vivo antifibrotisch und beeinflusst das Protease-Antiprotease-Gleichgewicht zugunsten des Matrixabbaus [2,67,73]. Bei Sklerodermie-Patienten führte eine Therapie mit rekombinantem humanem Relaxin über 6 Monate zu einer Verbesserung der Hautveränderungen und der Lungenfunktion [74].

\section{Zusammenfassung und Ausblick}

Der Therapieschwerpunkt chronisch-entzündlicher Krankheiten der Lunge lag in den vergangenen Jahrzehnten auf dem Einsatz immunsuppressiver Medikamente. Dieser Therapieansatz kann erfolgreich sein, wie z. B. bei Sarkoidose oder exogen-allergischer Alveolitis. Er kann aber auch versagen, wie man im Fall der IPF sieht. Die Forschung der letzten Jahre zeigte, dass chronische Erkrankungen nicht reine Entzündungsvorgänge sind, sondern chronische bzw. rezidivierende Entzündung kombiniert mit pathologischer Wundheilung. Zytokine sind in der Pathogenese maßgeblich involviert und ein attraktives Ziel für pharmakologische Interventionen. TGF $\beta$ hat als profibrotischer Wachstumsfaktor eine Schlüsselstellung und kann auf verschiedenen Wegen inaktiviert oder in seiner Wirkung abgeschwächt werden. Dazu sind sowohl „klassische“ Medikamente wie ACE-Hemmer oder Pirfenidon in der Lage als auch Immuntherapeutika wie Interferone. Eine Reihe multizentrischer prospektiver Studien wird derzeit durchgeführt. Es ist berechtigte Hoffnung erlaubt, dass sich das klinische Management fibrosierender Lungenerkrankungen in den kommenden Jahren bessern wird. 
${ }^{29}$ Tufvesson E, Westergren-Thorsson G. Alteration of proteoglycan synthesis in human lung fibroblasts induced by interleukin-1beta and tu-

${ }^{1}$ Lympany PA, du Bois RM. Diffuse lung disease: product of genetic susceptibility and environmental encounters. Thorax 1997; 52: 92 - 94

${ }^{2}$ Gross TJ, Hunninghake GW. Idiopathic pulmonary fibrosis. N Engl J Med 2001; 345: 517-525

${ }^{3}$ Ryu JH, Colby TV, Hartman TE. Idiopathic pulmonary fibrosis: current concepts. Mayo Clin Proc 1998; 73: 1085 - 1101

${ }^{4}$ American Thoracic Society. Idiopathic pulmonary fibrosis: diagnosis and treatment. International consensus statement. American Thoracic Society (ATS), and the European Respiratory Society (ERS). Am J Respir Crit Care Med 2000; 161: 646-664

${ }^{5}$ Selman M, King TE, Pardo A. Idiopathic Pulmonary Fibrosis: Prevailing and Evolving Hypotheses about Its Pathogenesis and Implications for Therapy. Ann Intern Med 2001; 134: 136-151

${ }^{6}$ Sime PJ, Gauldie J. Mechanisms of scarring. In: Evans TWHC (editor). ARDS in adults. London: Chapman \& Hall Medical, 1996: 215 - 231

${ }^{7}$ Sheppard D. Pulmonary fibrosis: a cellular overreaction or a failure of communication? J Clin Invest 2001; 107: 1501 - 1502

${ }^{8}$ Gauldie J, Kolb M, Sime PJ. A new direction in the pathogenesis of idiopathic pulmonary fibrosis? Respir Res 2002; 3: 1

9 Lasky JA, Brody AR. Interstitial fibrosis and growth factors. Environ Health Perspect 2000; 108 Suppl 4: $751-762$

${ }^{10}$ Allen JT, Spiteri MA. Growth factors in idiopathic pulmonary fibrosis: relative roles. Respir Res 2002; 3: 13

${ }^{11}$ Kunkel SL. Chemotactic cytokines: the chemokine family. In: Phan S (editor). Pulmonary fibrosis. New York: Marcel Dekker, 1995: 579-597

${ }^{12}$ Gauldie J, Sime PJ, Xing Z et al. Transforming growth factor-beta gene transfer to the lung induces myofibroblast presence and pulmonary fibrosis. Curr Top Pathol 1999; 93: 35-45

${ }^{13}$ Remick D. Cytokines and pulmonary fibrosis. In: Pulmonary fibrosis. New York: Marcel Dekker, 1995: 599-626

${ }^{14}$ Khalil N, O'Connor RN. Cytokine regulation of pulmonary fibrosis: TGFbeta. In: Pulmonary fibrosis. New York: Marcel Dekker, 1995: 627-645

${ }^{15}$ Keane MP, Strieter RM. The importance of balanced pro-inflammatory and anti-inflammatory mechanisms in diffuse lung disease. Respir Res 2002; $3: 5$

${ }^{16}$ Ward PA, Hunninghake GW. Lung inflammation and fibrosis. Am J Respir Crit Care Med 1998; 157: S123-S129

${ }_{17}$ Dinarello CA. Interleukin-1. Cytokine Growth Factor Rev 1997; 8: 253-265

${ }^{18}$ Rochester CL, Elias JA. Cytokines and cytokine networking in the pathogenesis in interstitial and fibrotic lung disorders. Semir Respir Crit Care Med 1994; 14: 389-416

${ }^{19}$ Boyle JE, Lindroos PM, Rice AB et al. Prostaglandin-E2 counteracts interleukin-1beta-stimulated upregulation of platelet-derived growth factor alpha-receptor on rat pulmonary myofibroblasts. Am J Respir Cell Mol Biol 1999; 20: $433-440$

${ }^{20}$ Phan SH, Kunkel SL. Lung cytokine production in bleomycin-induced pulmonary fibrosis. Exp Lung Res 1992; 18: 29-43

21 Thrall RS, Scalise PJ. Bleomycin. In: Phan SH (editor). Pulmonary Fibrosis. New York: Marcel Dekker, 1995: 231 - 292

22 Pan LH, Ohtani H, Yamauchi K et al. Co-expression of TNF alpha and IL-1 beta in human acute pulmonary fibrotic diseases: an immunohistochemical analysis. Pathol Int 1996; 46: 91 - 99

${ }^{23}$ Johnston CJ, Piedboeuf B, Rubin P et al. Early and persistent alterations in the expression of interleukin-1 alpha, interleukin-1 beta and tumor necrosis factor alpha mRNA levels in fibrosis-resistant and sensitive mice after thoracic irradiation. Radiat Res 1996; 145: 762 - 767

${ }^{24}$ Lee YM, Hybertson BM, Cho HG et al. Platelet-activating factor contributes to acute lung leak in rats given interleukin-1 intratracheally. Am J Physiol Lung Cell Mol Physiol 2000; 279: L75 - L80

${ }^{25}$ Hybertson BM, Lee YM, Repine JE. Phagocytes and acute lung injury: dual roles for interleukin-1. Ann NY Acad Sci 1997; 832: 266-273

${ }^{26}$ Kolb M, Margetts PJ, Anthony DC et al. Transient expression of IL-1beta induces acute lung injury and chronic repair leading to pulmonary fibrosis. J Clin Invest 2001; 107: 1529-1536

${ }^{27}$ Kotecha S, Wilson L, Wangoo A et al. Increase in interleukin (IL)- 1 beta and IL-6 in bronchoalveolar lavage fluid obtained from infants with chronic lung disease of prematurity. Pediatr Res 1996; 40: 250-256

${ }^{28}$ Sporn MB, Roberts AB. Peptide growth factors and their receptors. New York: Springer, 1991

mor necrosis factor-alpha. J Cell Biochem 2000; 77: 298 - 309
30 Ortiz LA, Lasky J, Hamilton Jr RF et al. Expression of TNF and the necessity of TNF receptors in bleomycin-induced lung injury in mice. Exp Lung Res 1998; 24: 721 - 743

${ }^{31}$ Sime PJ, Marr RA, Gauldie D et al. Transfer of tumor necrosis factor-alpha to rat lung induces severe pulmonary inflammation and patchy interstitial fibrogenesis with induction of transforming growth factorbeta1 and myofibroblasts. Am J Pathol 1998; 153: 825-832

32 Ziegenhagen MW, Schrum S, Zissel G et al. Increased expression of proinflammatory chemokines in bronchoalveolar lavage cells of patients with progressing idiopathic pulmonary fibrosis and sarcoidosis. J Investig Med 1998; 46: 223-231

33 O'Kane S, Ferguson MW. Transforming growth factor beta $\mathrm{s}$ and wound healing. Int J Biochem Cell Biol 1997; 29: 63 - 78

${ }^{34}$ Letterio JJ, Roberts AB. TGF-beta: a critical modulator of immune cell function. Clin Immunol Immunopathol 1997; 84: 244-250

${ }^{35}$ Munger JS, Huang X, Kawakatsu $\mathrm{H}$ et al. The integrin alpha v beta 6 binds and activates latent TGF beta 1 : a mechanism for regulating pulmonary inflammation and fibrosis. Cell 1999; 96: 319-328

${ }^{36}$ Fish JE, Peters SP. Airway remodeling and persistent airway obstruction in asthma. J Allergy Clin Immunol 1999; 104: 509-516

${ }^{37}$ Mutsaers SE, Bishop JE, McGrouther G et al. Mechanisms of tissue repair: from wound healing to fibrosis. Int J Biochem Cell Biol 1997; 29: $5-17$

38 Border WA, Noble NA. Transforming growth factor beta in tissue fibrosis. N Engl J Med 1994; 331: 1286 - 1292

${ }^{39}$ Rubin P, Johnston CJ, Williams JP et al. A perpetual cascade of cytokines postirradiation leads to pulmonary fibrosis [see comments] Int J Radiat Oncol Biol Phys 1995; 33: 99-109

${ }^{40}$ Sime PJ, Xing Z, Graham FL et al. Adenovector-mediated gene transfer of active transforming growth factor- beta 1 induces prolonged severe fibrosis in rat lung. J Clin Invest 1997; 100: 768-776

${ }^{41}$ Anscher MS, Kong FM, Andrews K et al. Plasma transforming growth factor beta1 as a predictor of radiation pneumonitis. Int J Radiat Oncol Biol Phys 1998; 41: 1029-1035

${ }^{42}$ Fabisiak JP, Kelley J. Platelet derived growth factor. In: Kelley J (editor). Cytokines of the lung. New York: Marcel Dekker, 1993: 3-39

${ }^{43}$ Rice AB, Moomaw CR, Morgan DL et al. Specific inhibitors of plateletderived growth factor or epidermal growth factor receptor tyrosine kinase reduce pulmonary fibrosis in rats. Am J Pathol 1999; 155: $213-221$

${ }^{44}$ Lasky JA, Tonthat B, Liu JY et al. Upregulation of the PDGF-alpha receptor precedes asbestos-induced lung fibrosis in rats. Am J Respir Crit Care Med 1998; 157: $1652-1657$

${ }^{45}$ Bostrom H, Willetts K, Pekny M et al. PDGF-A signaling is a critical event in lung alveolar myofibroblast development and alveogenesis. Cell 1996; 85: 863-873

${ }^{46}$ Chung KF, Barnes PJ. Cytokines in asthma. Thorax 1999; 54: 825-857

47 Barrios R, Pardo A, Ramos C et al. Upregulation of acidic fibroblast growth factor during development of experimental lung fibrosis. Am J Physiol 1997; 273: L451 - L458

${ }^{48}$ Liebler JM, Picou MA, Qu Z et al. Altered immunohistochemical localization of basic fibroblast growth factor after bleomycin-induced lung injury. Growth Factors 1997; 14: $25-38$

${ }^{49}$ Kadono T, Kikuchi K, Kubo M et al. Serum concentrations of basic fibroblast growth factor in collagen diseases. J Am Acad Dermatol 1996; 35: $392-397$

${ }^{50}$ Stern JB, Fierobe L, Paugam C et al. Keratinocyte growth factor and hepatocyte growth factor in bronchoalveolar lavage fluid in acute respiratory distress syndrome patients. Crit Care Med 2000; 28: 2326-2333

51 Anscher MS, Peters WP, Reisenbichler H et al. Transforming growth factor beta as a predictor of liver and lung fibrosis after autologous bone marrow transplantation for advanced breast cancer. $\mathrm{N}$ Engl J Med 1993; 328: $1592-1598$

52 Vogel E, Kolb M, Mueller J et al. BAL levels of the profibrotic cytokines TGFbeta and PDGF in patients with interstitial lung disease. Eur Respir J 2000; 14: 369S

53 Piguet PF, Vesin C. Treatment by human recombinant soluble TNF receptor of pulmonary fibrosis induced by bleomycin or silica in mice. Eur Respir J 1994; 7: 515-518 
54 Piguet PF, Vesin C, Grau GE et al. Interleukin 1 receptor antagonist (IL1ra) prevents or cures pulmonary fibrosis elicited in mice by bleomycin or silica. Cytokine 1993; 5: 57-61

${ }^{55}$ Entzian P, Zahringer U, Schlaak M et al. Comparative study on effects of pentoxifylline, prednisolone and colchicine in experimental alveolitis. Int J Immunopharmacol 1998; 20: $723-735$

${ }^{56}$ Zabel P, Entzian P, Dalhoff K et al. Pentoxifylline in treatment of sarcoidosis. Am J Respir Crit Care Med 1997; 155: 1665-1669

${ }^{57}$ Iyer SN, Gurujeyalakshmi G, Giri SN. Effects of pirfenidone on transforming growth factor-beta gene expression at the transcriptional level in bleomycin hamster model of lung fibrosis. J Pharmacol Exp Ther 1999; 291: $367-373$

${ }^{58}$ van den Berg WB. Anti-cytokine therapy in chronic destructive arthritis. Arthritis Res 2001; 3: $18-26$

${ }^{59}$ Wang $\mathrm{Q}$. Wang Y, Hyde DM et al. Reduction of bleomycin induced lung fibrosis by transforming growth factor beta soluble receptor in hamsters. Thorax 1999; 54: $805-812$

${ }^{60}$ Giri SN, Hyde DM, Braun RK et al. Antifibrotic effect of decorin in a bleomycin hamster model of lung fibrosis. Biochem Pharmacol 1997; 54: $1205-1216$

${ }^{61}$ Giri SN, Hyde DM, Hollinger MA. Effect of antibody to transforming growth factor beta on bleomycin induced accumulation of lung collagen in mice. Thorax 1993; 48: 959-966

${ }^{62}$ Kolb M, Margetts PJ, Galt T et al. Transient Transgene Expression of Decorin in the Lung Reduces the Fibrotic Response to Bleomycin. Am J Respir Crit Care Med 2001; 163: 770-777

${ }^{63}$ Molteni A, Moulder JE, Cohen EF et al. Control of radiation-induced pneumopathy and lung fibrosis by angiotensin-converting enzyme inhibitors and an angiotensin II type 1 receptor blocker. Int J Radiat Biol 2000; 76: $523-532$

${ }^{64}$ Gurujeyalakshmi G, Hollinger MA, Giri SN. Pirfenidone inhibits PDGF isoforms in bleomycin hamster model of lung fibrosis at the translational level. Am J Physiol 1999; 276: L311 - L318
${ }^{65}$ Gurujeyalakshmi G, Hollinger MA, Giri SN. Regulation of transforming growth factor-beta1 mRNA expression by taurine and niacin in the bleomycin hamster model of lung fibrosis. Am J Respir Cell Mol Biol 1998; 18 : $334-342$

${ }^{66}$ Ziesche R, Hofbauer E, Wittmann K et al. A preliminary study of longterm treatment with interferon gamma-1b and low-dose prednisolone in patients with idiopathic pulmonary fibrosis. N Engl J Med 1999; 341: $1264-1269$

${ }^{67}$ Franklin TJ. Therapeutic approaches to organ fibrosis. Int J Biochem Cell Biol 1997; 29: 79-89

68 Marshall RP, McAnulty RJ, Laurent GJ. Angiotensin II is mitogenic for human lung fibroblasts via activation of the type 1 receptor. Am J Respir Crit Care Med 2000; 161: 1999-2004

${ }^{69}$ Yoshida M, Sakuma-Mochizuki J, Abe K et al. In vivo gene transfer of an extracellular domain of platelet-derived growth factor beta receptor by the HVJ-liposome method ameliorates bleomycin-induced pulmonary fibrosis. Biochem Biophys Res Commun 1999; 265: 503 - 508

${ }^{70}$ Bienkowski RS, Gotkin MG. Control of collagen deposition in mammalian lung. Proc Soc Exp Biol Med 1995; 209: 118 - 140

${ }^{71}$ Gurujeyalakshmi G, Giri SN. Molecular mechanisms of antifibrotic effect of interferon gamma in bleomycin-mouse model of lung fibrosis: downregulation of TGF-beta and procollagen I and III gene expression. Exp Lung Res 1995; 21: $791-808$

72 Raghu G, Johnson WC, Lockhart D et al. Treatment of idiopathic pulmonary fibrosis with a new antifibrotic agent, pirfenidone: results of a prospective, open-label Phase II study. Am J Respir Crit Care Med 1999; 159: 1061 - 1069

${ }^{73}$ Unemori EN, Pickford LB, Salles AL et al. Relaxin induces an extracellular matrix-degrading phenotype in human lung fibroblasts in vitro and inhibits lung fibrosis in a murine model in vivo. J Clin Invest 1996; 98: $2739-2745$

${ }^{74}$ Seibold JR, Korn JH, Simms R et al. Recombinant human relaxin in the treatment of scleroderma. A randomized, double-blind, placebo-controlled trial. Ann Intern Med 2000; 132: 871 - 879 